\title{
EVOLUCIÓN DE LA DISTRIBUCIÓN DE LOS FORAMINÍFEROS BENTÓNICOS EN UN MEDIO CONTAMINADO: EL ESTUARIO DEL RÍO ODIEL (HUELVA, SO DE ESPAÑA)
}

\author{
María Luz GONZÁLEZ-REGALADO, Francisco RUIZ \\ MUÑOZ y José BORREGO FLORES
}

Departamento de Geología. Facultad de Ciencias Experimentales. Universidad de Huelva. 21819-Palos de la Frontera (Huelval)

\begin{abstract}
González-Regalado, M.L., Ruiz Muñoz, F. y Borrego Flores, J. 1996. Evolución de la distribución de los foraminíferos bentónicos en un medio contaminado: el estuario del río Odiel (Huelva, SO de España). [Evolution of the distribution of benthic foraminifera in a polluted area: the Odiel river estuary (Huelva. SW Spain]. Rerista Española de Paleontología, 11 (1), 1-10. ISSN 0213-6937.
\end{abstract}

\begin{abstract}
By studyng the benthic foraminifera of the Odicl estuary it has been possible to determine the inpact of some natural factors (hydrodynamics) and some man-made ones (metal pollution and by the dredging of sediments) on the various populations.

In the main channel of the upper estuary, the sum total of a high flow regime, the coarse grain of the sediments, high metal pollution and an acid $\mathrm{pH}$ maly caused the disappearance of these protists. Only some isolated sectors of salt marsh with an important organic content show appreciable fauna.

In the lower, marine estuary, two areas can be distinguished: the main channel, without fauna because of a high metal pollution and the permanent dredging of the river mouth, and the Punta Umbría sector with less polution and an appreciable faunal diversity. However, the diversity and abundance of individuals decreased because of changes in the sedimentary processes.
\end{abstract}

Keywords: Foraminifera, metal pollution, sedimentary processes, Odiel river, SW Spain.

\section{RESUMEN}

El estudio de los foraminíferos bentónicos del estuario del río Odiel (SO España) permite determinar el impacto de algunos factores naturales (hidrodinámica) y antrópicos (contaminación metálica y dragado) en las poblaciones de estos organismos.

En el canal principal del estuario alto, la coincidencia de una fuerte hidrodinámica, una textura gruesa de los sedimentos, alta polución y pH ácido ha provocado la desaparición de estos protistas.

En el estuario bajo y marino, pueden distinguirse 2 sectores: el canal principal, sin fauna debido a la altísima contaminación y el dragado permanente de la desembocadura del río; y el sector de Punta Umbría, con menos polución y una importante diversidad. En esta zona, el número de especies e individuos ha disminuido debido a cambios en los procesos sedimentarios.

Palabras clave: Foraminíferos, contaminación metálica, procesos sedimentarios, río Odiel, SO España.

\section{INTRODUCCIÓN}

El estudio de los foraminíleros bentónicos presentes en medios acuáticos actuales revela una importante información sobre la influencia de la hidrodinámica, sedimentación, contaminación y otros factores en los organismos vivos y su transporte post-mortem.

\section{Foraminíferos actuales de la Península Ibérica}

En España, investigaciones de estas características son comunes desde 1952. Colom (1952; 1974) realiza el estudio de los Foraminíteros de las rías de Galicia, costas cantábricas y litoral mediterráneo. Bilbao Moral y Lamolda (1982) mostraron la distribución de los foraminíferos actuales en las costas de Vizcaya, así como su relación con el sustrato. Pascual (1984; 1992) efectúa un análisis detallado de la microfauna (foraminíleros y ostrácodos) de las rías de Guernica y Bilbao, obteniendo interesantes resultados sobre el control de la salinidad, oxígeno disuelto, sustrato y metales pesados en sus asociaciones y especies. Cearreta (1986; 1988 a y b; 1989) describe la biocenosis y tanatocenosis de foraminíferos bentónicos en las rías y estuarios de 


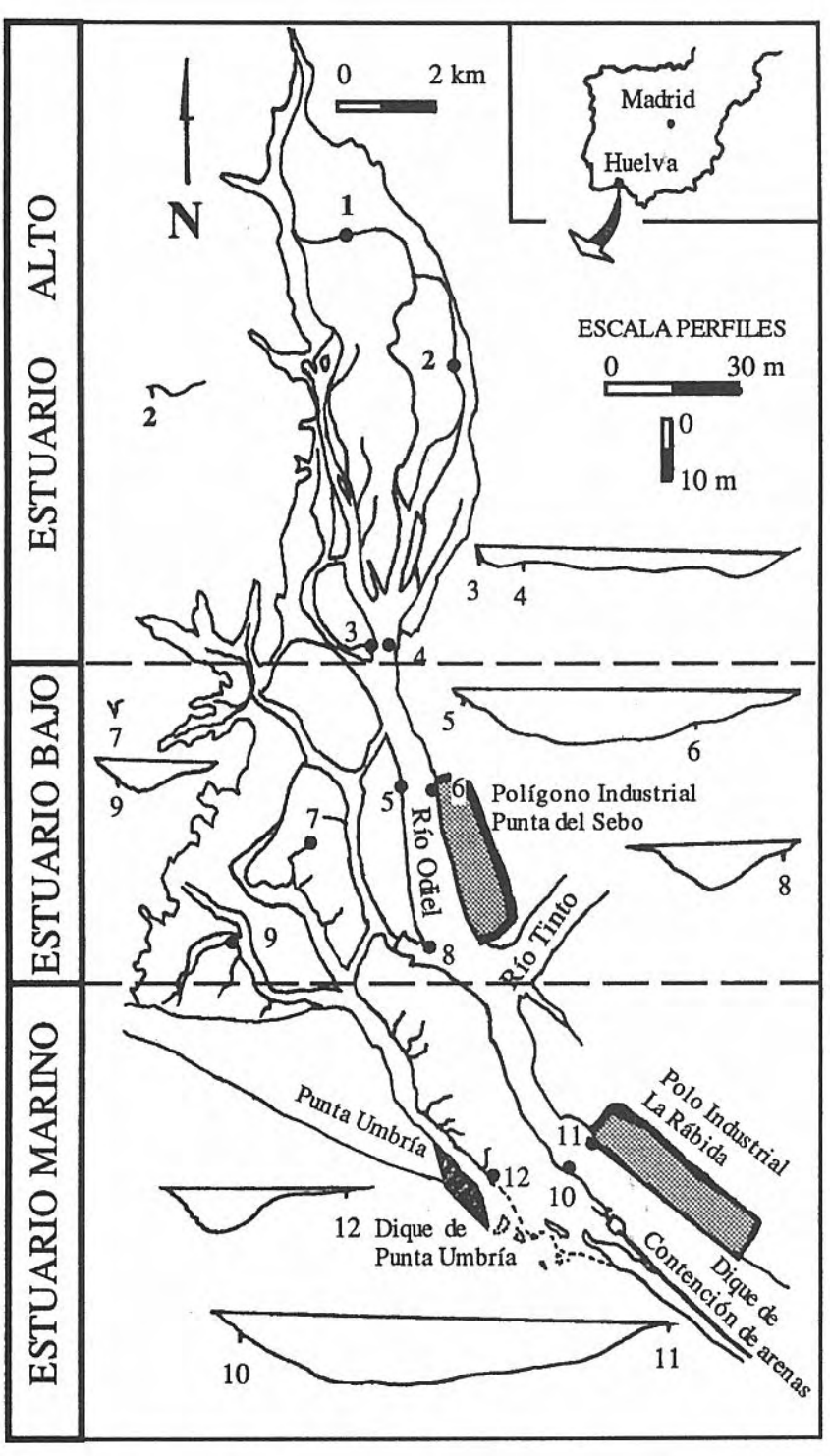

Figura 1. Situación geográfica del estuario del río Odiel. 112: Localización de los testigos estudiados.

Cantabria, así como la influencia de la salinidad y sustrato en los especímenes vivos. En el estuario del Bidasoa, Cearreta (1994) destaca la importancia de estas investigaciones en la reconstrucción de episodios transgresivos en rellenos estuarinos holocenos. En general, la biocenosis presenta una baja diversidad característica de estos medios de transición.

En el Mediterráneo, Mateu (1970) contribuye al conocimiento de la relación de estos protoctistas con diversos sustratos vegetales en Cataluña e Islas Baleares. Son reseñables los trabajos de Sánchez Ariza (1979; 1983; 1984) sobre los foraminíferos actuales de la zona nerítica del área Motril-Nerja, relacionando su distribución con la profundidad y el tipo de sedimentos (granulometría y contenido en carbonatos), entre otros factores. En el Puerto de Los Alfares (Tarragona), Sanchís et al. (1985) y Alberola et al. (1991) aportan datos relevantes sobre algunos aspectos tafonómicos que inciden en la dispersión de estos microorganismos.
Los foraminíferos de la región del Algarve (Portugal) fueron determinados por Galhano (1963). Más recientemente, Levy et al. (1993) ampliaron este estudio al resto del litoral portugués, deduciendo las asociaciones más importantes mediante técnicas estadísticas.

En el litoral suratlántico español, los primeros estudios similares son recientes. González-Regalado et al. (1990) detallan la distribución de foraminíferos y ostrácodos en la ría del Piedras, una zona no contaminada situada al $\mathrm{O}$ del sector aquí estudiado.

A nivel mundial, es destacable la recopilación de Murray (1991), que proporciona una visión muy completa sobre los aspectos ecológicos de los foraminíferos actuales en todo el mundo, incluyendo datos de las principales aportaciones en nuestro país.

\section{Foraminíferos y contaminación}

La influencia de la contaminación en foraminíferos es aún poco conocida. En áreas altamente polucionadas de Francia y España, los foraminíferos bentónicos están prácticamente ausentes (Philippe- Loeillet, 1983; Pascual, 1992). Anantha y Nigam (1984) observan efectos corrosivos, ornamentaciones degradadas, profundización de las suturas y ampliación de las aperturas en especies de medios contaminados de la costa occidental de la India.

En el presente trabajo, se estudia la evolución de los foraminíferos bentónicos en 12 testigos continuos seleccionados en distintos submedios del río Odiel (SO de España). Se comparan las asociaciones actuales de este medio y las formas subfósiles presentes antes y durante la época más importante de vertidos contaminantes en esta área (periodo 1966 - 1980).

\section{MARCO GEOGRÁFICO}

El río Odiel es una pequeña corriente fluvial que discurre por la provincia de Huelva durante $85 \mathrm{~km}$, desde su nacimiento en la Sierra de Aracena hasta la desembocadura en el Golfo de Cadiz. En la confluencia con el río Tinto, forma un estuario (Fig. 1) de marcado carácter mareal (tipo "Bar-buil Estuary" de Fairbridge, 1980).

En el interior, se desarrollan amplias llanuras mareales vegetadas, de origen mixto (continentalmarino). El cierre del estuario está constituido por una flecha litoral, considerablemente desarrollada de O a E en los últimos años (flecha de Punta Umbría), y por una sucesión de islas-barrera, entre las que se encuentran cuerpos de marisma salobre.

\section{MARCO HIDROLÓGICO}

\section{Río y mareas}

Los aportes fluviales del río Odiel presentan una enorme irregularidad interanual y estacional. Los meses 
de mayores aportes son diciembre, enero y febrero (en torno a los $100 \mathrm{Hm}^{3}$ ), mientras que durante julio y agosto no se llega a $1 \mathrm{Hm}^{3}$.

El régimen mareal es mesomareal, semidiurno con una desigualdad diaria leve (Borrego et al., 1992). El rango medio de las mareas es $2,10 \mathrm{~m}$, alcanzando los 3,50 $\mathrm{m}$ durante las mareas vivas medias y $0,70 \mathrm{~m}$ en las mareas muertas de equinocio. La onda de marea se desplaza por el interior del estuario a una velocidad entre 25 y $45 \mathrm{~km} / \mathrm{h}$, según un modelo de propagación hipersincrónico (Borrego, 1992).

La mezcla de aguas es controlada por el prisma mareal y la componente fluvial. Para mareas muertas/vivas muertas y caudales fluviales medios, el estuario del río Odiel se enmarca en los estuarios con "Estratificación Parcial" (Simmons, 1955). En los años muy secos, pueden aparecer condiciones hipersalinas estivales, que originan una cuña salina desde el estuario hacia la zona marina adyacente, durante los ciclos de bajamar (Borrego, op. cit.).

\section{Análisis del agua}

En 1981-1982, el Instituto Español de Oceanografía (I.E.O., 1982) y el M.O.P.U. (1982) efectuaron diversos muestreos en distintos puntos del cauce. En el estuario alto, se detectó una elevada acidez, así como altas concentraciones de sulfatos y zinc, procedentes de explotaciones mineras situadas al $\mathrm{N}$ de la provincia. También se obtuvieron importantes cantidades de fosfatos, atribuidas a fertilizantes agrícolas, además de una importante contaminación bacteriana, por vertidos de poblaciones próximas. En el estuario bajo y marino, el $\mathrm{pH}$ presentaba valores muy bajos (a veces inferiores a 5), interpretados como consecuencia de vertidos industriales o de la convergencia de las aguas ácidas de los ríos Tinto y Odiel, al no ser corregidas por la acción tamponadora del agua marina.

En abril de 1991, el I.E.O. (1992) determinó un pH casi neutro $(6,61-7,36)$ en los estuarios bajo y marino, durante la pleamar, tanto en el fondo como en la superficie. Estos valores disminuyeron ostensiblemente en la superficie del estuario durante la bajamar $(4,43)$, manteniéndose casi constantes en el fondo. Las altísimas concentraciones de nitratos (hasta $76 \mathrm{umol} / \mathrm{l}$ ) y fosfatos (hasta $170 \mathrm{umol} / \mathrm{l}$ ) se relacionaron con descargas de industrias fosforeras y los aportes fluviales.

En general, estas aguas están bien oxigenadas $(>6$ $\mathrm{mlg} / \mathrm{l})$, con máximos en abril. Sólo en los tránsitos pleamar-bajamar y viceversa, hay una disminución (5 $\mathrm{mlg} / \mathrm{l}$ ) en el contenido en $\mathrm{O}_{2}$ disuelto, para aumentar paulatinamente en el semiciclo mareal diario posterior (Borrego, 1992)

\section{ANÁLISIS TEXTURAL DE LOS SEDIMENTOS}

Borrego (op. cit.) analiza la textura de las muestras aquí estudiadas. Diferencia 3 zonas (Fig. 1) :

\section{Estuario alto}

En las zonas de canal y barra mareal, la interacción marea-río produce diversas distribuciones granulométricas, claramente diferenciadas en los testigos (Tabla 1). Fases de dominio de la componente fluvial ocasionan el depósito de arenas gruesas, escasamente bioturbadas, al $\mathrm{N}$ de este sector (testigo 1). Cerca del límite con el estuario bajo, este dominio se traduce en la aparición de arenas finas, con frecuentes depósitos residuales de conchas (testigo 4). Los periodos de máxima influencia mareal provocan la sedimentación de fangos ricos en materia orgánica, prontamente bioturbados (tramo medio del testigo 2).

En el borde de canal, no hay sedimentación actual. En la superficie se observa un sustrato arenoso consolidado con numerosos ostreidos (Crassostrea angulata). Los ejemplares se presentan en posición de vida, conservando las dos valvas. En la actualidad, no existe ninguna biocenosis similar en este sector del estuario.

La marisma salada presenta una alternancia característica de limos y arcillas limosas, altamente bioturbadas por halófitas (Spartina s. l.), con contenidos en materia orgánica superiores al $10 \%$ (tramo superior del testigo 3).

\section{Estuario bajo y zona interna del subsistema de Punta Umbría}

Las facies de canal están compuestas por limos arcillosos de color negro (testigos 6 y 8), con laminación paralela de escala centimétrica. Esta estructura puede quedar destruida por una importante bioturbación (poliquetos y bivalvos). Los restos de conchas son localmente importantes en el subsector de Punta Umbría (tramo inferior del testigo 9).

El borde de canal sólo alcanza cierta extensión areal en los márgenes del canal principal del río Odiel. Está constituido por arcillas limosas de color negro, con un importante grado de bioturbación (tramo superior del testigo 8). Son abundantes las galerías de crustáceos (Uca puxna en particular) y poliquetos (Arenicolides eucaudata, entre otros), ausentes en el borde de canal del estuario alto.

La marisma salada (testigo 5) presenta un tamaño medio de grano algo más fino que en el estuario alto, con una importante bioturbación por crustáceos y raíces que conlleva la desaparición de cualquier ordenamiento interno previo del sedimento. Un rasgo fisiográfico frecuente son los escarpes erosivos en sus bordes, originados por el oleaje y/o las corrientes mareales. Otras formas con presencia importante en las zonas de marisma alta son las charcas someras de carácter hipersalino ("pool marshes") y los esteros abandonados ("channel pans").

\section{Estuario marino y zona externa del subsistema de Punta Umbría}

El canal principal sufre un dragado continuo. Se depositan limos arcillosos negros (tramo superior del 


\begin{tabular}{|c|c|c|c|c|}
\hline MUESTRA & PROFUNDIDAD (cm) & CARACTERISTICAS & MEDIO & TASA (cm/año) \\
\hline 1.1 & SUPERACE & Arena gruesa no bioturbada. & Barra fluvial. & 1,2 \\
\hline 1.2 & 25 & Arena gruesa no bioturbada. & Barra fluvial. & \\
\hline 1.3 & 55 & Arena gruesa no bioturbada. & Barra fluvial. & \\
\hline 2.1 & SUPERACE & Arenas gruesas. Escasa bioturbación & Canal. Dominio fluvial. & 1,6 \\
\hline 2.2 & 20 & Fango. Bioturbación muy abundante. & Canal. Dominio mareal. & \\
\hline 2.3 & 35 & Arena gruesa no bioturbada & Canal. Dominio fluvial. & \\
\hline 3.1 & SUPERACE & Fango con raíces. Bioturbación muy importante. & Marisma. & 1,4 \\
\hline 3.2 & 28 & Arena gruesa no bioturbada. & Borde de canal. & \\
\hline 3.3 & 60 & Arena fina no bioturbada. & Borde de canal. & \\
\hline 4.1 & SUPERACE & Arenas finas. Depósito residual de conchas. & Canal-borde de canal. & 1,6 \\
\hline 4.2 & 30 & Arenas finas. Depósito residual de conchas. & Canal-borde de canal. & \\
\hline 4.3 & 60 & Arenas finas. Depósito residual de conchas. & Canal-borde de canal. & \\
\hline 5.1 & SUPERACE & Fango con raices. Bioturbación muy abundante. & Marisma. & 1,15 \\
\hline 5.2 & 30 & Fango con raíces y galerías. Bioturbación abundante. & Marisma. & \\
\hline 5.3 & 60 & Fango con galerías. Bioturbación importante. & Borde de canal. & \\
\hline 6.1 & SUPERACEE & Fango. Bioturbación importante. & Canal. & 2,3 \\
\hline 6.2 & 32 & Fango. Escasa bioturbación. & Canal. & \\
\hline 6.3 & 67 & Fango. Escasa bioturbación. & Canal. & \\
\hline 7.1 & SUPERACE & Fango con galerías. Bioturbación importante. & Borde de canal. & 1,7 \\
\hline 7.2 & 30 & Fango. Escasa bioturbación. & Canal finalizador. & \\
\hline 7.3 & 60 & Fango. Escasa bioturbación. & Canal finalizador. & \\
\hline 8.1 & SUPERACE & Fango con galerías. Bioturbación importante. & Borde de canal. & DRAGADO \\
\hline 8.2 & 35 & Fango con galerías. Bioturbación abundante. & Canal. & \\
\hline 8.3 & 65 & Fango. Escasa bioturbación. & Canal. & \\
\hline 9.1 & SUPERACE & Fango con galerías. Bioturbación importante. & Canal. & 1,76 \\
\hline 9.2 & 30 & Fango con galerías. Bioturbación importante. & Canal. & \\
\hline 9.3 & 65 & Fango. Escasa bioturbación. Conchas dispersas. & Canal. & \\
\hline 10.1 & SUPERACIE & Arena fina. & Canal. & DRAGADO \\
\hline 10.2 & 15 & Fango. Bioturbación importante. & Canal. & \\
\hline 10.3 & 30 & Arenas gruesas con gravas. Depósito residual de conchas. & Canal. & \\
\hline 11.1 & SUPERACIE & Fango con galerías. Bioturbación importante. & Canal. & DRAGADO \\
\hline 11.2 & 39 & Fango con galerías. Escasa bioturbación. & Canal. & \\
\hline 11.3 & 77 & Arena fina. & Canal. & \\
\hline 12.1 & SUPERACE & Fango con galerías. Bioturbación importante. Flaser. & Llanura mareal. & 1,54 \\
\hline 12.2 & 25 & Arena gruesa. Escasa bioturbación. Flaser. & Llanura mareal. & \\
\hline 12.3 & 50 & Arena gruesa. Escasa bioturbación. Flaser. & Llanura mareal. & \\
\hline
\end{tabular}

Tabla 1. Caracterización sedimentológica de los testigos estudiados.

testigo 11 y tramo medio del testigo 10), masivos o con laminación paralela. Los porcentajes medios en materia orgánica se aproximan al $15 \%$. En las zonas más profundas y cercanas a la desembocadura, aparecen arenas medias a gruesas, con algunos restos de conchas y gruesos cantos de cuarzo muy redondeados.

El borde de canal tiene similares características al descrito en el estuario bajo, con mayores contenidos en arena y mayor diversidad en la morfología de las galerías. Las acumulaciones de conchas y algunas colonias de fanerógamas (Zostera marina y Zostera noltii) son también frecuentes en las zonas topográficas más bajas de este borde de canal.

Existen 2 tipos diferentes de marisma salada. La marisma alta, situada en la parte posterior de los ganchos más antíguos, presenta rasgos similares a los descritos en la marisma del estuario bajo. En las marismas más jóvenes del sistema, es característica la alternancia de capas de limos arenosos y arenas limosas, con un $10 \%$ medio de materia orgánica.

La llanura mareal próxima a Punta Umbría (testigo 12) está formada por arenas limosas mal clasificadas, de color negro, con alto porcentaje orgánico (15 a $32 \%$ ). Las galerías están producidas por diversas especies de poliquetos (sobre todo Arenicolides ecaudata y Nereis diversicolor), crustáceos y moluscos.

\section{CONTENIDO EN METALES PESADOS}

\section{Acción antrópica histórica y reciente}

El río Odiel atraviesa una de las provincias metalíferas más importantes de Europa, con grandes yacimientos de pirita y sulfuros masivos de metales básicos $(\mathrm{Cu}, \mathrm{Pb}, \mathrm{Zn})$. El abandono de escombreras en sus márgenes (consecuencia de una minería milenaria), así como su lavado por la escorrentía superficial, ha provocado el vertido al cauce de notables cantidades de metales pesados. Cerca de las minas más importantes (Riotinto, La Zarza, Sotiel), se alcanzan valores elevados en $\mathrm{Pb}, \mathrm{Cu}$ y $\mathrm{Zn}$ (Requena et al., 1991). Una parte es conducida en suspensión hacia el estuario, donde se acumula especialmente en los sedimentos más finos.

A mediados de la década de los 60, se inicia la construcción de dos Polos de Promoción Industrial (Fig. 1) cerca de la desembocadura, formados por industrias químicas básicas (fertilizantes, cloroalcalinas, etc). Sus 
vertidos ácidos incontrolados, con importantes contenidos en metales pesados, provocaron un progresivo deterioro del ecosistema estuarino. Entre estos elementos contaminantes, el $\mathrm{Cu}, \mathrm{Pb}$ y $\mathrm{Zn}$ son considerados como muy tóxicos (Wood, 1973), ya que son muy accesibles y entran a formar parte de compuestos organometálicos ligados a los nutrientes (Forstner, 1983). Se introducen en la cadena trófica de estos medios y son difícilmente reciclables por los organismos, acumulándose en los tejidos.

Diversos estudios efectuados sobre el contenido en metales pesados de los tejidos blandos de bivalvos avalan esta pauta. En ejemplares de Chamelea gallina recogidos en la desembocadura de los ríos Tinto y Odiel, la Agencia del Medio Ambiente (A.M.A., 1991) confirmó la presencia de concentraciones de $\mathrm{Cu}$ cercanas al doble permitido (20 ppm, según el B.O.E. de 6 de Junio de 1985), así como concentraciones superiores de $\mathrm{Ni}$ al resto de los ejemplares del litoral onubense. En esta especie, la acumulación de $\mathrm{Cu}$ provoca en ocasiones una coloración azul clara en la concha (González-Regalado, 1994).

En los últimos años, se han puesto en marcha diversas medidas correctoras. Desde 1988, la Agencia del Medio Ambiente (A.M.A. 1988-1993) desarrolla un plan de inspección de las variables físico-químicas de las aguas y sedimentos de la zona, para intentar conseguir un inicio de recuperación ecológica. A ello se une una importante transformación en los procesos de producción de las empresas químicas, cuyo Plan Corrector ha disminuido los vertidos industriales ácidos desde las $29.960 \mathrm{~T}$ anuales registradas en 1989 a las 1.704 T de 1993 (Junta de Andalucía, 1994).

También es destacable el impacto de diversas construcciones en este sector. En 1980 finalizó la construcción del dique de contención de arenas del Puerto de Huelva (Fig. 1), que supuso un obstáculo al tránsito sedimentario. Ello ha modificado el modelo de circulación mareal en el interior del estuario, provocando un aumento de los espacios marismeños (Tabla 1: testigos 3 y 5 ) y el cierre progresivo del medio estuarino. Actualmente, esta construcción se revela ineficaz debido a la acción eólica sobre las playas adosadas al S del dique, que introduce arena en el canal principal y hace necesario su continuo dragado.

\section{Evolución de la contaminación metálica}

Desde la época tartésica, los desechos mineros han provocado el vertido al cauce de elementos contaminantes. Parte de esta polución ha quedado reflejada en los sedimentos finos del estuario del río Odiel, auténticas trampas de metales. Antes de la construcción de los Polos de Promoción, se encuentran importantes concentraciones de $\mathrm{Zn}(>2.000 \mathrm{ppm})$ en los sedimentos del canal principal y borde de canal del río Odiel, así como en la marisma del subsector de Punta Umbría. Algo similar fue detectado por Borrego (1992) en la base de testigos tomados en el estuario fluvial bajo,

\begin{tabular}{|c|c|c|c|c|c|}
\hline ESTUARIO ALTO & $\mathrm{Cr}$ (ppm) & $\mathrm{Cu}$ (ppm) & $\mathrm{Zn}(\mathrm{ppm})$ & $\mathrm{Pb}(\mathrm{ppm})$ & TiO2 (\%) \\
\hline \multicolumn{6}{|l|}{ MARISMA } \\
\hline$<1966(70 \mathrm{~cm})$ & 101 & 19 & 86 & 23 & 0,83 \\
\hline 1991 & 85 & 952 & 2280 & 557 & 0,72 \\
\hline \multicolumn{6}{|l|}{ CANAL-BORDE CANAL } \\
\hline$<1966(70 \mathrm{~cm})$ & 73 & 35 & 123 & 32 & 0,79 \\
\hline $1966-1980(30 \mathrm{~cm})$ & & 710 & 1400 & 175 & \\
\hline 1991 & 149 & 2990 & 2740 & 1930 & 0,66 \\
\hline \multicolumn{6}{|l|}{ ESTUARIO BAJO } \\
\hline \multicolumn{6}{|l|}{ A) CANAL PRINCIPAL ODIEL } \\
\hline \multicolumn{6}{|l|}{ MARISMA } \\
\hline$<1966(50 \mathrm{~cm})$ & 112 & 115 & 135 & 66 & 0,88 \\
\hline $1966-1980(25 \mathrm{~cm})$ & 101 & 1270 & 2680 & 413 & 0,6 \\
\hline 1991 & 82 & 1680 & 1270 & 1510 & 0,57 \\
\hline \multicolumn{6}{|l|}{ CANAL-BORDE CANAL } \\
\hline$<1966(70 \mathrm{~cm})$ & 87 & 1100 & 2260 & 289 & 1,09 \\
\hline $1966-1980(40 \mathrm{~cm})$ & 106 & 1480 & 2750 & 738 & 0,72 \\
\hline \multicolumn{6}{|l|}{$1989^{* *}-1991$} \\
\hline 1) Entre las muestras 5 y 6 & $157^{* *}$ & $2248^{* *}$ & $4007^{* *}$ & $2404^{* *}$ & 0,76 \\
\hline 2) Muy proximo a la muestra 8 & $273^{* *}$ & $3453^{* *}$ & $4538^{* *}$ & $2691^{* *}$ & 0,57 \\
\hline \multicolumn{6}{|l|}{ B) SECTOR PUNTA UMBRIA } \\
\hline \multicolumn{6}{|l|}{ MARISMA } \\
\hline$<1966(80 \mathrm{~cm})$ & 82 & 121 & 242 & 36 & 0,81 \\
\hline 1991 & 85 & 1020 & 2790 & 497 & 0,71 \\
\hline \multicolumn{6}{|l|}{ CANAL-BORDE CANAL } \\
\hline$<1966(72 \mathrm{~cm})$ & 97 & 386 & 2280 & 81 & 0,84 \\
\hline 1991 & 115 & 2000 & 1160 & 1780 & 0,66 \\
\hline \multicolumn{6}{|l|}{ ESTUARIO MARINO } \\
\hline \multicolumn{6}{|l|}{ A) CANAL PRINCIPAL ODIEL } \\
\hline \multicolumn{6}{|l|}{ MARISMA } \\
\hline 1991 & 165 & 2130 & 2890 & 1170 & 0,82 \\
\hline \multicolumn{6}{|l|}{ CANAL } \\
\hline 1975 & & $1400^{*}$ & $3100^{*}$ & $1600^{*}$ & \\
\hline \multicolumn{6}{|l|}{$1989^{* *}-1991$} \\
\hline 1) Confluencia Tinto-Odiel & $146^{* *}$ & $4765^{* *}$ & 2600 ** & $4086^{* *}$ & \\
\hline 2) Entre las muestras 10 y 11 & $120^{* *}$ & $5360^{* *}$ & $6156^{* \star}$ & $3754^{\star *}$ & 0,76 a 0,82 \\
\hline \multicolumn{6}{|l|}{ B) SECTOR PUNTA UMBRIA } \\
\hline \multicolumn{6}{|l|}{ MARISMA } \\
\hline$<1966(60 \mathrm{~cm})$ & 60 & 202 & 1620 & 51 & 0,71 \\
\hline 1991 & 58 & 304 & 529 & 137 & 0,81 \\
\hline \multicolumn{6}{|l|}{ CANAL-BORDE CANAL } \\
\hline 1991 & 79 & 575 & 1437 & 364 & 0,7 \\
\hline \multicolumn{6}{|l|}{ LLANURA MAREAL } \\
\hline$<1966(40 \mathrm{~cm})$ & 20 & 83 & 156 & 19 & 0,3 \\
\hline $1966-1975(20 \mathrm{~cm})$ & 71 & 420 & 2700 & 129 & 0,7 \\
\hline 1991 & 36 & 130 & 323 & 53 & 0,55 \\
\hline
\end{tabular}

Tabla 2. Evolución del contenido en metales pesados de los sedimentos del estuario del río Odiel (en ppm, excepto $\mathrm{TiO}_{2}$, en \%). * Stenner y Nickless (1975); ** Cabrera et al. (1992); resto: Borrego (1992).

con elevados contenidos en $\mathrm{Pb}$ (hasta $4.860 \mathrm{ppm}$ ), $\mathrm{Zn}$ (1.850 ppm) y Cu (hasta $1.530 \mathrm{ppm})$.

Durante el periodo 1966-1980, los vertidos incontrolados producen un incremento notable de metales en todos los sectores del estuario, llegando a multiplicarse por 20 las concentraciones previas de $\mathrm{Cu}$ (marisma del canal principal). Elementos relativamente fijos, como el $\mathrm{Pb}$, confirman esta tendencia. La ausencia de barreras físicas permite la introducción de $\mathrm{Cu}, \mathrm{Pb}$ y $\mathrm{Zn}$ en el subsistema de Punta Umbría, acumulándose preferentemente en la llanura mareal (Tabla 2).

En los últimos años, la construcción del dique de contención de arenas del Puerto de Huelva ha aislado parcialmente este subsistema, registrándose una notable disminución de la contaminación metálica en la llanura mareal y la marisma salobre. En el resto del estuario, han aumentado todos los contenidos metálicos, consecuencia 
de una corrección aún parcial de los vertidos industriales y la redistribución de sus componentes metálicos por procesos mareales.

\section{ANÁLISIS DE LOS, FORAMINÍFEROS BENTÓNICOS}

\section{Metodología}

La campaña de muestreo a testigo continuo se realizó en abril de 1991. En cada testigo seleccionado, se separaron 3 muestras, cada una de $39,27 \mathrm{~cm}^{3}$. Según la tasa de sedimentación observada (Borrego, 1992), la profundidad de la muestra basal se situó entre 40 y $80 \mathrm{~cm}$ de la superficie. La muestra intermedia, correspondiente al intervalo 1966-1980, se obtuvo entre los 20 y $40 \mathrm{~cm}$, en tanto que la última muestra corresponde a los $2 \mathrm{~cm}$ superiores del sedimento. A esta muestra se le añadió una solución de formol y Rosa de Bengala para la conservación e identificación de los ejemplares vivos (método de Walton, 1952). Este método de tinción, generalmente aceptado para la determinación de la biocenosis, muestra un índice de error muy elevado (Sánchez Ariza, com. per.), y debe ser complementado con la observación detallada de las alteraciones en los caracteres externos del caparazón (roturas, erosiones, disoluciones, etc).

\section{Evolución de los foraminíferos bentónicos: análisis y discusión}

En las Tablas 3, 4 y 5, se cuantifican los resultados obtenidos para los 3 periodos seleccionados $(<1966$; 1966-1980 y 1991).

\section{A) Estuario alto}

En las barras fluviales (testigo 1), sólo existen prolongados periodos de inmersión durante las mareas medias y vivas. Esta elevada exposición subaérea, unida a una notable acidez de las aguas y un tamaño de grano excesivamente grosero, han impedido el desarrollo de los

\begin{tabular}{|c|c|c|c|c|c|c|c|c|c|c|c|c|}
\hline ESPECIES/MUESTRAS & 1 & 2 & 3 & 4 & 5 & 6 & 7 & 8 & 9 & 10 & 11 & 12 \\
\hline Ammonia inflata & 1 & & & 11 & & & 6 & 88 & 94 & 44 & 93 & 624 \\
\hline Ammonia ammoniformis & & & & 1 & & & & & 1 & & & \\
\hline Ammonia beccarii & & & & & & & 6 & 4 & & 6 & 3 & \\
\hline Anomalinoides sp. & & & & & & & & 5 & & & & \\
\hline Astacolus sp. & & & & & & & & & & & & 3 \\
\hline Astrononion stelligerum & & & & & & & 6 & 10 & 76 & 4 & 3 & 148 \\
\hline Bolivina spathulata & & & & & & & & & 2 & 3 & 3 & 10 \\
\hline Bolivina sp. & & & & & & & & & & & & 1 \\
\hline Bulimina costata & & & & & & & & & & & & 3 \\
\hline Bulimina elongata? & & & & & & & & & & & & 7 \\
\hline Bulimina sp. & & & & & & & & & 6 & & & 3 \\
\hline Cancris auriculus & & & & & & & & & & & 1 & \\
\hline Cassidulina carinata & & & & & & & & & & & & 3 \\
\hline Cibicides pseudoungerianus & & & & & & & & & 1 & & & \\
\hline Cribroelphidium vadescens & & & & & & & 7 & 2 & & & 20 & \\
\hline Elphidium advenum & & & & & & & 1 & 3 & & 4 & 5 & \\
\hline Elphidium crispum & & & & & & & & & 2 & 5 & 13 & 25 \\
\hline Elphidium flexuosum & & & & & & & & & & 1 & & \\
\hline Elphidium williamsoni & & & & 1 & & & 3 & 6 & 10 & 1 & & 53 \\
\hline Elphidium sp. & & & & & & & & & & & & 6 \\
\hline Eponides antillarum & & & & & & & & & & & 3 & \\
\hline Florilus boueanum & & & & & & & & & & 3 & & \\
\hline Globobulimina auriculata & & & & & & & & & & & & 1 \\
\hline Globobulimina sp. & & & & & & & & & & & & 3 \\
\hline Globulina sp. & & & & & & & & & & 1 & & \\
\hline Haynesina depressula & & & & & & & 1 & & 1 & & & 3 \\
\hline Haynesina germanica & & & & & & & 3 & 11 & 22 & 1 & & \\
\hline Hanzawaia boueana & & & & & & & & & 1 & & & \\
\hline Heterolepa bellincionii & & & & & & & & & & & 13 & \\
\hline Heterolepa sp. & & & & & & & & & & 1 & & 26 \\
\hline Jadammina macrescens & & & & & & & & & 1 & & & \\
\hline Lagena costata & & & & & & & & & 1 & & & \\
\hline Neoconorbina williamsoni & & & & & & & 1 & & & 14 & 5 & 104 \\
\hline Nonion elongatum & & & & & & & & & 3 & 3 & 3 & \\
\hline Nonion incrassatum & & & & & & & 3 & & 26 & & & 7 \\
\hline Oolina faveolata & & & & & & & & & 1 & & & \\
\hline Planorbulina mediterranensis & & & & & & & & & 1 & & & 52 \\
\hline Protelphidium granosum & & & & & & & 2 & & & 2 & & \\
\hline Quinqueloculina padana & & & & & & & & & & 6 & & \\
\hline Quinqueloculina seminulum & & & & & & & & & & 1 & 5 & 8 \\
\hline Rosalina globularis & & & & & & & & & 3 & 3 & 5 & 70 \\
\hline Stomatorbina concentrica & & & & & & & & & & 2 & 3 & \\
\hline Triloculina oblonga & & & & & & & & & & 2 & & \\
\hline Trochammina inflata & & & & & 2 & & & & & & & \\
\hline Uvigerina costata & & & & & & & & & 1 & & & \\
\hline $\mathrm{N}^{2}$ INDIVIDUOS & 1 & 0 & 0 & 13 & 1 & 0 & 39 & 129 & 253 & 107 & 178 & 1160 \\
\hline$N^{2}$ ESPECIES & 1 & 0 & 0 & 3 & 1 & 0 & 11 & 8 & 19 & 20 & 15 & 21 \\
\hline
\end{tabular}

Tabla 3. Distribución y abundancia de las especies de foraminíferos bentónicos del estuario del río Odiel antes de 1966.

foraminíferos bentónicos en este medio. Sólo aparecen esporádicos caparazones de Ammonia inflata (Tabla 3), con erosiones provocadas por el transporte mareal.

En el canal principal y borde de canal (testigos 2, 4 y muestras subfósiles del testigo 3), no aparecen estos

\section{Lámina I}

1. Trochammina inflata (Montagu). Muestra 5. Vista dorsal.

2. Trochammina inflata (Montagu). Muestra 5. Vista umbilical.

3. Quinqueloculina oblonga (Montagu). Muestra 10.

4. Quinqueloculina seminulum (Linné). Muestra 9.

5. Stomatorbina concentrica (Parker y Jones). Muestra 9.

6. Rosalina globularis D’Orbigny. Muestra 12. Vista dorsal.

7. Rosalina globularis D’Orbigny. Muestra 12. Vista umbilical.

8. Neoconorbina williamsoni (Chapman y Parr). Muestra 12. Vista dorsal.
9. Neoconorbina williamsoni (Chapman y Parr). Muestra 12. Vista umbilical

10. Planorbulina mediterranensis D'Orbigny. Muestra 12. Vista dorsal.

11. Elphidium crispum (Linné). Muestra 9.

12. Elphidium macellum (Fichtel y Moll). Muestra 9.

13. Elphidium williamsoni Haynes. Muestra 12.

14. Astrononion stelligerum (D'Orbigny). Muestra 9.

15. Haynesina germanica (Ehrenberg). Muestra 9.

16. Haynesina germanica (Ehrenberg). Muestra 9. Vista apertural.

Escala $=50 \mu$ 
Lámina I

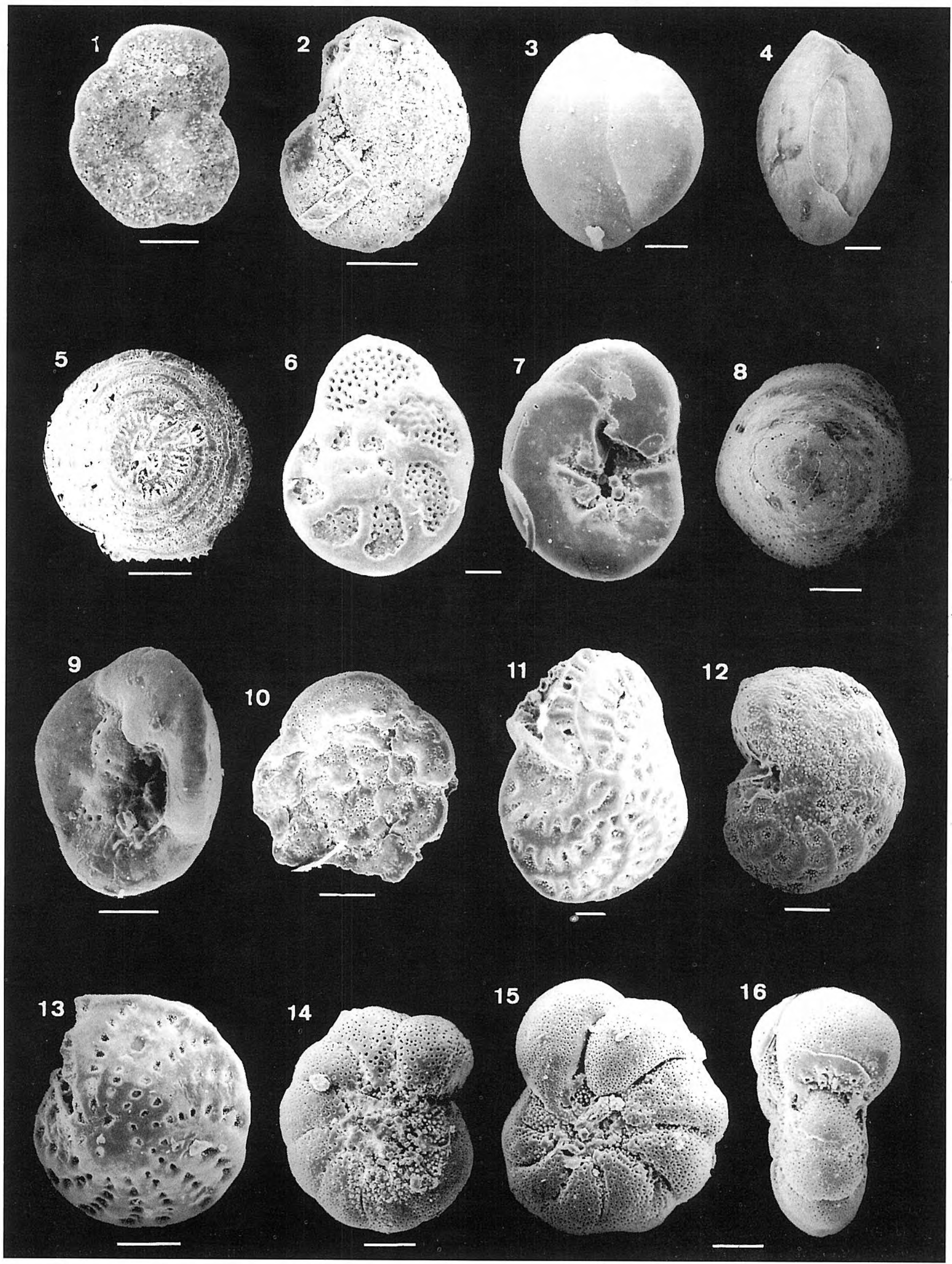

Revista Española de Paleontología, 11(1), 1996. 


\begin{tabular}{|l|c|c|c|c|c|c|c|c|c|c|c|c|}
\hline ESPECEESMUESTRAS & 1 & 2 & 3 & 4 & 5 & 6 & 7 & 8 & 9 & 10 & 11 & 12 \\
\hline Ammonia inflata & & & 1 & & & & 158 & & 37 & & & 7714 \\
\hline Astrononion stelligerum & & & & & & & 83 & & 20 & & & 2394 \\
\hline Bolivina spathulata & & & & & & & & & & & & 1064 \\
\hline Bulimina elongata subulata & & & & & & & & & & & & 798 \\
\hline Cribroelphidium vadescens & & & & & & & 269 & & 10 & & & \\
\hline Elphidium crispum & & & & & & & & & & & & 665 \\
\hline Elphidium macellum & & & & & & & 8 & & 1 & & & 1463 \\
\hline Elphidium oceanensis & & & & & & & & & & & & 399 \\
\hline Elphidium williamsoni & & & & & & & 19 & & 3 & & & 3591 \\
\hline Florilus boueanum & & & & & & & & & & & & 133 \\
\hline Haynesina germanica & & & & & & & & & & & & 1064 \\
\hline Heterolepa bellincionii & & & & & & & & & & & & 133 \\
\hline Jadammina macrescens & & & 1 & & & & & & & & & \\
\hline Lagena striata & & & & & & & 1 & & & & & \\
\hline Neoconorbina williamsoni & & & & & & & & & & & & 3857 \\
\hline Planorbulina mediterranensis & & & & & & & & & & & & 2128 \\
\hline Rosalina globularis & & & & & & & & & & & & 1995 \\
\hline Quinqueloculina jugosa & & & & & & & & & & & & 266 \\
\hline Quinqueloculina oblonga & & & & & & & & & & & & 266 \\
\hline Quinqueloculina seminulum & & & & & & & & & & & & 266 \\
\hline & & & & & & & & & & & & \\
\hline N2 INDIVIDUOS & 0 & 0 & 2 & 0 & 0 & 0 & 538 & 0 & 72 & 0 & 0 & 28296 \\
\hline N2ESPECIES & 0 & 0 & 2 & 0 & 0 & 0 & 6 & 0 & 6 & 0 & 0 & 17 \\
\hline
\end{tabular}

Tabla 4. Distribución y abundancia de las especies de foraminíferos bentónicos del estuario del río Odiel entre 1966 y 1980.

organismos (Tablas 3, 4 y 5). Estos canales son poco profundos (Fig. 1) y el lecho queda expuesto durante las bajamares extremas si la descarga fluvial es pequeña. Este estrés hidrodinámico, unido a una fuerte contaminación metálica, son condiciones adversas para los microorganismos (Carbonel, 1980; Pascual, 1992). Se han observado ejemplares resedimentados en las muestras anteriores a 1966 y en los sedimentos recientes. La acidez de las aguas durante el periodo 1966-1980 habría condicionado la disolución de las formas transportadas durante este periodo (Tabla 4).

La dinámica sedimentaria reciente ha provocado la aparición de nuevas extensiones de marisma en este sector (parte superficial del testigo 4). Su relativo aislamiento, unido a un menor contenido metálico, permite la presencia de una reducida biocenosis de especies estuarinas típicas (Tabla 5), como Ammonia inflata , Ammonia beccarii , Astronion stelligerum (Lám. 1, fig. 14) y Cribroelphidium vadescens (Tabla 5).

\section{B) Estuario bajo}

En el canal principal próximo al Polígono Industrial Punta del Sebo (testigo 6), el dragado continuo, elevadísimo contenido metálico y la fuerte hidrodinámica explican la ausencia de microfauna en todas las muestras.

Cerca de la confluencia con el río Tinto (testigo 8), los foraminíferos bentónicos eran frecuentes antes de 1966. Entre las especies autóctonas más representativas, aparecían $A$. inflata, A. stelligerum y Haynesina germanica (Lám. 1, figs. 15 y 16). El efecto de la polución fue drástico, observándose su total desaparición posteriormente. Actualmente, sólo se encuentran formas rotas y/o erosionadas.

Las marismas saladas recientemente creadas (testigo 5) encierran una biocenosis formada por Trochammina inflata (Lám. 1, figs. 1 y 2) y esporádicos ejemplares de Jadammina macrescens. Es una asociación típica de estos medios en otros estuarios europeos (Murray, 1991).

\begin{tabular}{|c|c|c|c|c|c|c|c|c|c|c|c|c|}
\hline ESPECIES / MUESTRAS & 1 & 2 & 3 & 4 & 5 & 6 & 7 & 8 & 9 & 10 & 11 & 12 \\
\hline Ammonia ammoniformis & & & & $6^{*}$ & & & 8 & $1^{*}$ & & & & \\
\hline Ammonia beccarii & & & 6 & & $1^{*}$ & & 5 & & 3182 & $4^{*}$ & & 83 \\
\hline Ammonia inflata & & & 45 & & & & & & 533 & $2^{*}$ & $3^{*}$ & 40 \\
\hline Anomalinoides sp. & & & & $2^{*}$ & & & & & $4^{*}$ & & & \\
\hline Astrononion stelligerum & & & 4 & & & & 7 & $1^{*}$ & 126 & $1^{*}$ & & 70 \\
\hline Cribroelphidium vadescens & & & 6 & & & & & & & & & \\
\hline Elphidium advenum & & & $5^{*}$ & & & & & & & & & $2^{*}$ \\
\hline Elphidium crispum & & & & & & & & & $10^{*}$ & $2^{*}$ & $3^{*}$ & \\
\hline Elphidium macellum & & & & & & & & & 198 & & & 67 \\
\hline Elphidium williamsoni & & & & $1^{*}$ & & & & & 33 & 1 & & 54 \\
\hline Haynesina germanica & & & & $1^{*}$ & & & 12 & & 33 & & & \\
\hline Jadammina macrescens & & & & & 12 & & & & & & & \\
\hline Lenticulina sp. & & & & & & & & & $1^{*}$ & & & \\
\hline Neoconorbina williamsoni & & & & & & & & & 9 & & & 17 \\
\hline Planorbulina mediterranensis & & & & & & & & & & & & 22 \\
\hline Quinqueloculina oblonga & & & & & & & & & & $2^{*}$ & & \\
\hline Quinqueloculina seminulum & & & & & & & & & $9^{*}$ & $1^{*}$ & & $8^{\circ}$ \\
\hline Rosalina globularis & & & & & & & $1^{*}$ & & & $1^{*}$ & & $27^{*}$ \\
\hline Stomatorbina concentrica & & & & & & & & & $8^{*}$ & & & \\
\hline Trochammina inflata & & & & & 286 & & & & & & & \\
\hline $\mathrm{N}^{2}$ INDIVIDUOS & 0 & 0 & 66 & 10 & 299 & 0 & 33 & 2 & 4146 & 14 & 6 & 390 \\
\hline NRESPECIES & 0 & 0 & 5 & 4 & 3 & 0 & 5 & 2 & 12 & 8 & 2 & 10 \\
\hline
\end{tabular}

Tabla 5. Distribución y abundancia de las especies de foraminíferos bentónicos del estuario del río Odiel en 1991. * Especies con evidencias de transporte.

En los canales finalizadores del subsistema de Punta Umbría (testigo 7), la progresiva colmatación y contaminación son factores limitantes para los microorganismos. La asociación inicial estaba formada por numerosos ejemplares de pocas especies (A. inflata, A. stelligerum, Cribroelphidium vadescens). En el periodo de máxima polución, este medio estaba ocupado por escasos ejemplares de estas especies, junto a otras formas estuarinas ( $H$. germanica, Elphidium williamsoni). Actualmente, el tránsito al borde de canal produce una disminución importante en el número de individuos.

En el canal distribuidor de Punta Umbría (testigo 9), los efectos de la polución han sido evidentes. La rica diversidad inicial, con mezcla de formas estuarinas y marinas transportadas, disminuyó drásticamente entre 1966 - 1980 (Tablas 3 y 4). Desde esta fecha, se ha acentuado el aislamiento de esta zona respecto a la fuente contaminante, debido a la construcción del dique de contención de arenas. Ello ha contribuido a una espectacular recuperación de los foraminíferos, con $A$. beccarii, A. inflata, A. stelligerum, Elphidium macellum (Lám. 1, fig. 12), E. williamsoni y H. germanica como componentes mayoritarios de la biocenosis actual.

\section{C) Estuario marino}

En el canal principal del río Odiel (testigos 10 y 11), existía una importante población de foraminíferos bentónicos autóctonos, junto a formas marinas resedimentadas (Tabla 3). La microfauna era más diversa en la ribera occidental y más numerosa en la zona oriental. El influjo de los vertidos industriales provocó la desaparición de la microfauna (Tabla 4). Actualmente, la presencia de formas transportadas sin evidencias de disolución indicaría una menor acidez en el fondo del canal respecto al periodo anterior.

En la llanura mareal de Punta Umbría (testigo 12), los foraminíferos bentónicos eran abundantes, con numerosas 
especies salobres y marinas resedimentadas (Planorbulina mediterranensis, Rosalina globularis, Quinqueloculina spp.). Parecida diversidad se observa en algunos estuarios actuales del N de España (Cearreta, 1989). Debido a su relativo aislamiento, la contaminación no afectó de forma significativa a esta zona, con un aumento enorme en el número de individuos entre 1966 y 1980, si bien redujo levemente el número de especies. Actualmente, el avance del spit de Punta Umbría impide la entrada de arena a esta zona y limita la acción del oleaje. El cambio de sedimentación (arenas a limos) ha inducido una disminución espectacular en el número de individuos, a pesar de una considerable merma en la contaminación metálica (Tabla 5). Este último efecto es consecuencia directa de la construcción del dique del Puerto de Huelva en 1980, que protege esta zona de la influencia fluvial directa.

\section{CONCLUSIONES}

El estudio de los foraminíferos bentónicos se revela como un método eficaz para medir la influencia de la. dinámica sedimentaria y la contaminación industrial en el estuario del río Odiel.

Antes de 1966, el canal principal comprendido entre los dos Polos de Promoción Industrial presentaba una variada microfauna ( A. inflata, A. stelligerum, $C$. vadescens, etc). La época de máxima contaminación coincidió con la total desaparición de estas especies e incluso de las formas resedimentadas. Actualmente, sólo se encuentran escasos ejemplares transportados.

En el subsistema de Punta Umbría, la contaminación afectó principalmente a las zonas más internas. Desde la construcción del dique de contención de arenas del Puerto de Huelva, se ha producido una notable recuperación en los canales distribuidores. En canales finalizadores, la progresiva colmatación conlleva una disminución drástica en diversidad y número de individuos.

Los efectos sedimentarios de la construcción del dique del Puerto de Huelva han sido considerables para la microfauna. El aumento en extensión de las marismas saladas ha propiciado la instalación de las especies típicas de este medio relativamente protegido en estuarios europeos no contaminados.

El desarrollo progresivo de la flecha de Punta Umbría produce unas condiciones poco favorables en la llanura mareal cercana. La sombra hidrodinámica creada, con cambios bruscos en el tipo de sedimento depositado, ha provocado una disminución importante en la diversidad y la instalación de una biocenosis típica de ambientes estuarinos restringidos.

\section{AGRADECIMIENTOS}

Expresamos nuestro agradecimiento a D. E. Gómez Asencio, del Instituto de Agrobiología y Recursos Naturales del C.S.I.C. de Sevilla, por la realización de las fotografías de M.E.B. A D. J. C. Fernández Caliani, por su colaboración en la composición del manuscrito. A los revisores, por sus aportaciones y consejos para la mejora del manuscrito.

Este trabajo ha sido financiado por la Dirección General de Investigación Científica y Técnica, proyecto PB93-1205, y por la Junta de Andalucía, P.A.I. 4070.

\section{BIBLIOGRAFÍA}

Alberola, C.,Usera, J. y García-Forner, A. 1991. Estructura de las tanatocenosis de foraminíferos arenáceos en el Puerto de los Alfaques (Tarragona). Revista Española de Paleontología, número extraordinario, 77-86.

A.M.A. 1988-1993. Plan de Policía de Aguas. Informes finales.

A.M.A. 1991 . Determinación del contenido de pesticidas en aguas y de metales en organismos vivos. Documento final. $86 \mathrm{pp}$.

Anantha, M. G. and Nigam, R. 1984. Benthic foraminifera as pollution indices in the marine environment of west coast of India. Rivista Italiana di Paleontologia e Stratigrafía, 89 (3), 421-436.

Bilbao Moral, G. y Lamolda, M. A. 1982. Resultados preliminares del estudio de los foraminíferos del litoral vizcaíno. Actas Primer Simposio Ibérico de estudios del bentos marino, 1-16.

Borrego, J. 1992. Sedimentología del estuario del río Odiel (Huelva, SO España). Tesis Doctoral Universidad de Sevilla, $311 \mathrm{p}$.

Borrego, J., Morales, J.A. y Pendón, J.G. 1992. Formación de sulfuros de hierro en estuarios actuales: algunas consideraciones genéticas. Geogaceta, 11, 125-127.

Cabrera, F., Conde, B. and Flores, V. 1992. Heavy metals in the surface sediments of the tidal river Tinto (SW Spain). Fresenius Environmental Bulletin, 1, 400-405.

Carbonel, P. 1980. Les ostracodes et leur intéret dans la définition des écosystèmes éstuariens et de plateforme continentale. Essais d'application à des domaines anciens. Mémoires de l'Institut de Géologie du Bassin d'Aquitaine, 11, 1-350

Cearreta, A. 1986. Distribution and ecology of benthic Foraminifera in the Rías of Santoña and San Vicente de la Barquera (Spain). Ph. D. Thesis University of Exeter. $307 \mathrm{pp}$.

Cearreta, A. 1988a. Distribution and ecology of benthic Foraminifera in the Santoña estuary, Spain. Revista Española de Paleontología, 3, 23-38.

Cearreta, A. 1988b. Population dinamics of benthic Foraminifera in the Santoña estuary, Spain. Revue de Paléobiologie, 2, 721-724.

Cearreta, A. 1989. Foraminiferal assemblages in the Ría of San Vicente de la Barquera (Cantabria, Spain). Revista Española de Micropaleontología, 21 (1), 67-80.

Cearreta, A. 1994. Análisis micropaleontológico e interpretación paleoecológica del relleno holoceno en el estuario del Bidasoa (Golfo de Bizkaia). Geobios, 27 (3), 271-283.

Colom, G. 1952. Foraminíferos de las costas de Galicia. Boletin del Instituto Español de Oceanografía, 55, 1-44.

Colom, G. 1974. Foraminíferos ibéricos. Introducción al 
estudio de las especies bentónicas recientes. Investigaciones Pesqueras, 38 (1), 1-245.

Fairbridge, P. V. 1980. The estuary: its definitions and geodynamic cycle. In: Chemistry and biochemystry of estuaries (Eds. E. Olausson and I. Cato), 1-32.

Forstner, U. 1983. Metal concentrations in River, Lake and Ocean Water. In: Metal Pollution in the Aquatic Environment Springer-Verlag Ed., 71-109.

Galhano, M. H. 1963. Foraminiferos da costa de Portugal (Algarve). Publicacao Instituto Zoologia Augusto Nobre. Facultad Ciencia Porto, 110 pp.

González-Regalado, E. 1994. Estudio de metales en Moluscos Bivalvos de la costa suratlántica andaluza. Tesis Doctoral (inéd.) Universidad de Sevilla. 300 pp.

González-Regalado, M. L., Ruiz Muñoz, F., Borrego, J. y Pendón, J.G. 1990. La microfauna de la Ría del Piedras (Huelva): algunos aspectos ambientales. Actas I Reunión sobre el Medio Ambiente en Andalucía , 274-280.

I.E.O. 1982 . Estudio de la contaminación de la ría de Huelva. Informe final. $199 \mathrm{pp.}$

I.E.O. 1992. Informe técnico $n^{\circ} 138:$ Variación espaciotemporal de parámetros físico-químicos y biológicos en la ría de Huelva y área de influencia, en el período 1987-1991. 103 pp.

Junta de Andalucía 1994. Medio Ambiente. 35 pp.

Levy, A., Mathieu, R., Poignant, A., Rosset-Moulinier, M., Ubaldo, M. de L. and Ambroise, D. 1993. Recent foraminifera from the continental margin of Portugal. Micropaleontology, 39 (1), 75-87.

Mateu, G. 1970. Estudio sistemático y bioecológico de los foraminíferos vivientes de los litorales de Cataluña y Baleares. Trabajos del Instituto Español de Oceanografía, 38, 1-84.

Murray, J. W. 1991. Ecology and palaeoecology of benthic Foraminifera. John Wiley \& Sons Ed. 396 pp.

M.O.P.U. 1982. Estudio de la contaminación de la ría de Huelva. Evaluación de vertidos, planes, usos y actividades en la zona de influencia. Diagnosis de la situación actual de marismas y esteros. Informe técnico, $378 \mathrm{pp}$.

Pascual, A. 1984. Los Foraminíferos actuales del litoral vizcaino. $K O B I E, \mathbf{1 4}, 341-350$.
Pascual, A. 1992. Utilización de los Foraminíferos bentónicos para un mejor conocimiento del medio ambiente en los estuarios vizcaínos (Gernika y Bilbao). Revista española de Micropaleontología, 25(1), 33-57.

Philippe-Loeillet, S. 1983. Contribution à l'étude des foraminiferes benthiques de zones soumises à la pollution urbaines (Emissaires des villes de Marseille et Toulon-Ouest) (Rade Abril de Toulon). Thèse Doctorat Université d'Aix-Marseille II, 126 pp.

Requena, A. A., Clauss, F. L. y Fernández-Caliani, J. C. 1991. Mineralogía y aspectos geoquímicos de los sedimentos actuales del río Odiel (Huelva). Cuadernos do Laboratorio Xeoloxico de Laxe, 16, 135-144.

Sánchez Ariza, M. C. 1979 . Estudio sistemáticoecológico de los foraminíferos recientes de la zona litoral Motril-Nerja. Tesis Doctoral Universidad de Granada, 213 pp.

Sánchez Ariza, M. C. 1983. Specific associations of recent benthic foraminifera of the neritic zone in the MotrilNerja area, Spain, as a function of depth: diversity and constancy. Journal of Foraminiferal Research, 13 (1), 13-20.

Sánchez Ariza, M. C. 1984. Especies bentónicas recientes de foraminíferos del sistema nerítico Motril-Nerja, España, con índice máximo de constancia en relación con la profundidad. Thalassas, 2, 7-12.

Sanchís, E., Ferré, E.J. y Usera, J. 1985. Aportación al conocimiento de los foraminíferos del Puerto de los Alfares (Tarragona: España). Abstracts VII Bienal Real Sociedad Española de Historia Natural, 136.

Simmons, H. B. 1955. Some effectsupland dischange on estuarine hydraulics. Proceedings of the American Society of Civil Engineers, 81, 1-20.

Stenner, R. D. and Nickless, G. 1975. Heavy metals in organism of the Atlantic coast of SW Spain and Portugal. Marine Pollution Bulletin, 6, 89-92.

Walton, W. R. 1952. Techniques for recognition of living foraminifera. Contribution from the Cushman Foundation for Foraminiferal Research, 3, 56-60.

Wood, J. M. 1973. Metabolic cycles for toxic elements in aqueous systems. Revista Internacional de Oceanografía Médica, 31-32, 7-17. 